This item was submitted to Loughborough's Research Repository by the author.

Items in Figshare are protected by copyright, with all rights reserved, unless otherwise indicated.

\title{
Ultra-efficient, widely tunable gold nanoparticles-based fiducial markers for X-ray imaging
}

\section{PLEASE CITE THE PUBLISHED VERSION}

http://dx.doi.org/10.1039/C6NR07021C

\section{PUBLISHER}

(c) Royal Society of Chemistry

\section{VERSION}

NA (Not Applicable or Unknown)

\section{PUBLISHER STATEMENT}

This work is made available according to the conditions of the Creative Commons Attribution-NonCommercialNoDerivatives 4.0 International (CC BY-NC-ND 4.0) licence. Full details of this licence are available at: https://creativecommons.org/licenses/by-nc-nd/4.0/

\section{LICENCE}

CC BY-NC-ND 4.0

\section{REPOSITORY RECORD}

Maiorano, G., Elisa Mele, C. Frassanito, E. Restini, Athanassia Athanassiou, and P.P. Pompa. 2016. "Ultraefficient, Widely Tunable Gold Nanoparticles-based Fiducial Markers for X-ray Imaging”. Loughborough University. https://hdl.handle.net/2134/23303. 


\title{
Ultra-efficient gold nanoparticles-based fiducial markers for X-ray imaging with 60-fold contrast enhancement
}

\author{
G. Maiorano ${ }^{1}$, E. Mele ${ }^{2,3}$, C. Frassanito ${ }^{4}$, E. Restini ${ }^{4}$, A. Athanassiou ${ }^{2}$, and P.P. Pompa ${ }^{1 *}$
}

1) Nanobiointeractions\&Nanodiagnostics, Istituto Italiano di Tecnologia (IIT), Via Morego 30, 16163, Genoa (Italy).

2) Smart Materials, Nanophysics Department, Istituto Italiano di Tecnologia (IIT), Via Morego 30, 16163, Genoa (Italy).

3) Department of Materials, Loughborough University, Leicestershire LE11 3TU, United Kingdom.

4) $X X X$, Bari (Italy).

\begin{abstract}
We show the development of a new class of highly efficient, biocompatible fiducial markers for Xray imaging and radiosurgery, based on polymer shells encapsulating engineered Au nanoparticle (NP) suspensions. Our smart fabrication strategy enable wide tunability of fiducial size, shape, and X-ray attenuation performance, up to record values $>20000$ Hounsfield units (HU), which are even higher than bulk gold. We report a nanoscale enhancement factor of ca. 60 -fold with respect to the bulk material, which allows superior imaging performance both in vitro and in vivo, strong stability over time and absence of classical "streak artefacts" of standard bulk fiducials. NP fiducials were probed in vivo, showing exceptional contrast efficiency, even after 2 weeks post-implant in mice.
\end{abstract}


Thanks to several recent technological breakthroughs, such as intensity-modulated radiotherapy (IMRT) and image-guided radiotherapy (IGRT), radiosurgery represents one of the most promising approaches for cancer treatment, achieving impressive spatial resolution for selective and efficient targeting and killing of tumor tissue [1-3]. Such technologies enable higher doses in target volumes of complex shape, while reducing toxicity to the surrounding healthy tissues and/or radiationsensitive organs. Radiosurgery locates the target site with high precision, relying on skull bony landmarks or on the spine (e.g. in case of intracranial lesions or spinal injuries, respectively) or on implanted radiopaque fiducial markers for all other applications involving soft tissues (e.g. lung, liver, pancreas, gastro-intestinal tract) [7-11], which are completely radio transparent and do not offer any reference point. Such fiducials, usually millimeter-sized cylinders made of pure gold, create an internal coordinate system that allows real-time tracking of the tumor position during radiation and precise alignment of the beams. However, the implantation of fiducial markers is an invasive procedure that may result in severe side effects, including inflammation, bleeding, infection, and pneumothorax [12-15]. This partially weakens the great advantages of radiosurgery, including its inherent non-invasive tumor killing properties.

In this work, we developed a low-cost, versatile alternative to standard gold fiducials that can be easily and safely implanted, exploiting engineered gold nanoparticles (AuNPs) properly encapsulated in polymeric spheres. Our innovative NP fiducials can reach extremely high X-ray imaging contrast with Hounsfield numbers (H.U.) higher than 20000, i.e. even better than bulk gold [Ref]), thanks to a nanoscale enhancement factor of ca. 60-fold with respect to the bulk material. We show that such innovative NP fiducials can be exploited in vivo, guarantying exceptional contrast efficiency, even after 2 weeks post-implant. Our fiducial markers were fabricated by encapsulating a small volume $(\sim 1-3 \mu \mathrm{l})$ of highly concentrated AuNPs dispersion in polydimethylsiloxane (PDMS) shells, while preserving the colloidal stability. Gold is characterized by a high X-rays absorption efficiency $\left(5.16 \mathrm{~cm}^{2} / \mathrm{g}\right.$ at $\left.100 \mathrm{keV}\right)$ with respect to iodinated agents (1.94), and water/soft tissues (0.17)[Ref. NIST]. Notably, AuNPs have demonstrated good potential as contrast agents in X-ray imaging [20-23+Nanomedicine+Adv Funct Mater 2014 and 2015], although quite limited absorption efficiencies have been reported to date in the literature, with typical $\mathrm{HU}<1000$ (while bulk gold has average $\mathrm{HU} \approx 5000$ and bone tissue $H U \approx 1000-2000$ ). Moreover, to the best of our knowledge, possible differences in the X-ray interaction mechanisms between nanoscale particles and bulk materials have not yet been observed and analyzed. In this work, we have investigated highly concentrated AuNP suspensions $(>100 \mathrm{mg} / \mathrm{ml})$, optimizing the whole fabrication process of our hybrid fiducials, so to guarantee their long-term colloidal stability. This enabled us to obtain, for the first time, much higher X-ray contrast efficiencies (HU $>>1000)$, providing evidence of a large enhancement of the absorption efficiency of nanoparticles with respect to bulk gold.

Spherical and monodispersed AuNPs with $70 \mathrm{~nm}$ diameter were synthesized as previously reported [Ref]. AuNPs were then stabilized by adding an aqueous solution of 2-[4-(2-hydroxyethyl)-1piperazinyl] ethane-sulfonic acid (HEPES) at $\mathrm{pH}$ 7.4. HEPES is an organic zwitterionic buffering agent effective in the physiological $\mathrm{pH}$ range from 6.8 to $8.2\left(\mathrm{pK}_{\mathrm{a}} 7.55\right)$, and it is widely used in biology/biochemistry due to its non-toxicity [28]. After HEPES stabilization, AuNPs were concentrated by applying several centrifugation cycles, and subsequently added of glycerol in order to obtain the optimal degree of viscosity and density. The AuNPs were carefully characterized by means of dynamic light scattering (DLS), Z-potential, transmission electron microscopy (TEM), and UV-vis absorption spectroscopy (Figure S1). AuNPs showed spherical morphology with size dispersion $<10 \%$. Accordingly, their absorption band was narrow, exhibiting the expected plasmon absorption maximum (Fig. S1b). DLS analysis (Fig. S1c) indicates well-suspended monodispersed NPs, with no detectable aggregation phenomena after concentration and glycerol addition. Moreover, Z-potential analysis shows that HEPES is a good capping agent that promotes the electrostatic stabilization of the colloids dispersion by impairing a strong negative surface charge to the NPs (Fig. S1d). 
The fabrication procedure of the hybrid NP fiducial markers is schematically shown in Fig. 1. Briefly, the PDMS base (part A, monomer) was mixed with the cross-linker (part B) at 9:1 ratio (A:B), stirred, degassed, and poured into a Petri dish. A volume (1-3 $\mu \mathrm{l})$ of AuNPs dispersion with the desired concentration was allocated inside the liquid pre-polymer by using a positive displacement micropipette. Thanks to the immiscibility and difference in surface tension between the aqueous AuNPs dispersion and the hydrophobic silicone pre-polymer ( $>65 \mathrm{dyn} / \mathrm{cm})$, the AuNPs drops acquired and kept a spherical shape in the PDMS matrix. The PDMS-AuNP system was cured at $60{ }^{\circ} \mathrm{C}$ for ... hours in an oven. After the complete polymerization of the polymer matrix, the hybrid NP fiducial markers were modelled to obtain single prototype devices, as showed in Figure $1 \mathrm{~b}$. The effective curing of silicone elastomers containing aqueous drops strongly depends on the interfacial interactions, dispersion composition, and potential impurities. During the curing process, at the interface between the silicone elastomer and the NPs, low molecular weight volatile substances and side reaction products were generated [REF], representing a potential source of both polymerization inhibition and AuNPs destabilization, with consequent irreversible aggregation. As shown in Fig. 2b, the fiducial markers obtained by this method exhibited a well-defined spherical surface without un-polymerized regions, indicating that the curing process was successful and that the AuNP dispersions did not act as inhibitor of the curing process. Importantly, during the polymerization of PDMS, AuNPs remained well dispersed in the aqueous solution without any agglomeration/aggregation phenomena, as demonstrated by UV-vis absorption spectroscopy (Fig. 2c). The peculiar localized surface plasmon resonance (LSPR) band, whose position and intensity depend on NP size, shape, concentration, and dispersion state, did not change significantly before and after encapsulation. Such strong particle stability in the fiducials was observed also over several months of monitoring at room conditions. The presence of HEPES as capping agent creates a strong negatively charged double layer onto the NP surface that electrostatically stabilizes the colloidal dispersion. Importantly, this molecule also acts as a slightly basic buffer that prevents destabilization of AuNPs upon release of by-products during the polymer curing process, such as acidic compounds. In preliminary experiments performed under identical conditions but with different stabilizing agents of AuNPs (including citrate, PVP, and serum proteins), in fact, we were not able to maintain NPs stability after polymer curing, regardless of NPs concentration, indicating that AuNPs and polymer curing were not compatible. HEPES stabilization was therefore our key smart solution to guarantee particle stability at highest concentrations along with protection from acids (and other curing byproducts), with consequent reliability of the whole fabrication process. It is also important to underline the presence of glycerol in the dispersion, which has a multifold role: it protects NPs against aggregation induced by heating (it prevents water evaporation in in vivo conditions at $37^{\circ} \mathrm{C}$ ) and provides the dispersion with proper density and viscosity, so to efficiently contrast the spontaneous NPs sedimentation over long-term (as mentioned above, the NP fiducials are in fact stable for months at room conditions).

The choice of PDMS was driven by its widely accepted biocompatibility (Generally Recognized As Safe, GRAS) and FDA approval for long-term medical implants [Ref], according to SP class VI. The key features relevant for our applications included flexibility, physiological indifference, excellent resistance to biodegradation and ageing, proper protection of the AuNPs suspension, and high biocompatibility.[19]

An important characteristic of our fiducial markers is the possibility to tune both their size and Xrays contrast efficiency, independently or simultaneously, thanks to the versatility and simplicity of the fabrication process. Fiducial size can be modulated by changing only the NPs drop volume inserted into the polymer, while X-rays contrast efficiency can be varied by changing the initial NPs concentration. This latter property can be tuned in a very wide range, without having limitations in the encapsulation of very concentrated NP suspensions. This gives extremely efficient fiducials, with unprecedented X-ray absorption efficiency (as it will be discussed later on).

As model prototypes, fiducial markers with diameters of 1.2 and $1.8 \mathrm{~mm}$ were fabricated by encase 0.90 or $3 \mu \mathrm{l}$ of AuNPs dispersion in the PDMS matrix, respectively. The effectiveness of these 
hybrid NP fiducials was evaluated by X-ray computed tomography (CT) imaging. CT scans were first taken in tissue equivalent phantoms. Representative images are shown in Fig. 2, with the corresponding HU numbers recorded (HU units are defined as the relative difference in linear attenuation coefficient between the sample and water). The ability of these fiducial markers to deliver CT images of great quality is evident. Notably, the achieved contrast of the NP fiducials is comparable and even superior to standard markers made of bulk gold (up to a record of $>20,000$ $\mathrm{HU}$ ). Such huge contrast values are much higher than previously reported, and highlight the possibility to precisely modulate the attenuation efficiency, as a function of the application, by fine tuning the AuNPs concentration. Furthermore, unlike standard fiducials that displays the classical "streak or void artifacts" that emanate radially outward from the fiducial [Huang, Phys Med Biol. 56, 5287 (2011)] (see Fig. 2, right panel), due to XXX effects [Ref], our NP fiducials do not show any imaging artifacts, at the same HU contrast, likely because...[explain]. This is an important finding for clinical applications. The absence of streak artifacts, in fact, may allow radiation oncologists to improve the treatment planning, avoiding the delivery of sub-optimal treatments because of uncertainties in targeting the total volume of interest.

The NP fiducials were also tested to evaluate their long-term stability, finding no appreciable decrease in X-ray contrast after 1 month at ambient conditions. Moreover, when maintained at 37 ${ }^{\circ} \mathrm{C}$ (in water), mimicking in vivo conditions, only a slight decrease ( $\left.\sim 10-15 \%\right)$ of contrast efficiency was observed after 2 weeks. This can be ascribed to the stabilizing effect of glycerol that helps preventing evaporation of the aqueous solvent through the microporous walls of PDMS. In fact, in absence of glycerol, the same fiducials displayed a significantly shorter performance, due to particle agglomeration and consequent drastic reduction of HU numbers.

Beyond the excellent versatility of these devices, thanks to HU spanning a very wide range, it is worth analyzing a novel aspect disclosed by the NP fiducials, namely the high nanoscale enhancement of X-ray attenuation with respect to the bulk material. In Fig. 3, we plotted the measured HU of the different NP fiducials as a function of NP concentration. A very good linear correlation was found $\left(\mathrm{R}^{2}=0.96\right)$, with $\mathrm{HU}$ steadily increasing with particle concentration, in line with the expected behavior at macroscale. However, from the direct comparison of the X-ray attenuation obtained with a bulk gold fiducial $\left(\mathrm{HU}_{\max } \approx 6000-8000\right)$ and with a NP fiducial of same efficiency, we found a nanoscale enhancement factor of ca. 60 -fold. This is because the same attenuation can be obtained with ca. 60 -fold less material (the density of bulk gold is ca. $20 \mathrm{~g} / \mathrm{ml}$ versus $0.3 \mathrm{~g} / \mathrm{ml}$ of the NP fiducial of identical HU numbers). It is noteworthy that, in the case of the NP fiducials, $8000 \mathrm{HU}$ attenuation can be achieved by an equivalent gold amount as low as $\approx 300$ $\mu \mathrm{g}$, versus ca. X g of a bulk gold fiducial (of Xmm x X mm dimensions), with a consequent difference in terms of gold mass of X-fold. To our knowledge, the observation of such nanoscale enhancement of X-ray attenuation is novel, so the explanation of the underlying mechanism is challenging. A qualitative explanation may rely on the strong role played by the huge surface area of AuNPs per mass unit, which likely increases the gold absorption cross section. In this X-ray energy range $(\sim 100 \mathrm{keV})$, the main interaction mechanism responsible for attenuation is the photoelectric effect... (qui se riusciamo a dire qualcosa di più in termini fisici)

The role of NPs surface is supported by experimental data. When the NPs in the fiducial core were destabilized (e.g., by excess heating), leading to particle aggregation (so reducing the total surface area), a dramatic decrease of X-ray attenuation efficiency occurred, because aggregated NPs rapidly approach the behavior of macroscale gold. In absence of surface enhancement, the HU numbers strongly drop down and the attenuation reaches the levels of the surrounding background. Hence, the dispersion status of the AuNPs is crucial for real applications, so we had to carefully optimize our fabrication procedure to guarantee long-term stability of the colloidal suspensions, also in in vivo conditions.

We also assessed the performance of the NP fiducials as a function of NP size (in the 50-120 nm range). In line with previous literature, we did not observe differences for the various sizes, when 
fiducials were directly compared in terms of same gold concentration [25, check], thus indicating that X-ray attenuation of AuNP dispersions is independent of the particle diameter in this size range. Moreover, in case of bigger particles (90-120 nm), we observed some sedimentation of the particle suspension over time, due to the high density of gold, which partly overcomes the ability of the charged capping agent and glycerol to keep nanoparticles well-dispersed.

As a final yet crucial point, we evaluated the stability and effectiveness of the NP fiducials in vivo, by implanting them in mice. We used fiducials fabricated with the maximum AuNP concentration (ca. $850 \mathrm{mg} / \mathrm{mL}$ ), in order to evaluate their in vivo performance in the most critical conditions (in terms of colloidal stability). On the other side, we aimed at analyzing in vivo the range of maximum X-rays attenuation achievable. NP fiducials were implanted in different organs of mice (including liver, stomach, spleen, scrotum, muscle), and both planar (whole animal) and axial CT images were acquired (representative images are reported in Fig. 4). As clearly shown in the two whole animal images, the NP fiducials offered an impressive X-ray attenuation, which was much higher than that displayed by all mouse bones. The same performance was confirmed by analyzing different animal sections, and HU numbers ranging around 20,000 units were recorded. Such high contrast values were never reported in vivo in previous studies, as we achieved a contrast improvement higher than 1 order of magnitude. Although such performance is not directly required in most current imaging applications, it is important to assess that the NP fiducials efficiently work in vivo also in extreme conditions, while new clinical applications might arise based on such novel characteristics. Furthermore, the huge contrast guaranteed by these NP fiducials may allow the use of significantly lower X-ray doses in clinical imaging, with clear benefit to the patients. Equally importantly, the NP fiducials did not show in vivo the same big streak artifacts that are typical of bulk fiducials. Only some effect is detectable at the highest HU numbers, though it can be strongly reduced at lower AuNP concentrations (as also shown above in Fig. X). Finally, we observed that the NP fiducials were stable in vivo over time, a crucial characteristic in real clinical applications. We found substantial stability of their attenuation efficiency after 1 week post-implant, and a minor ( $15-20 \%)$ decrease after 2 weeks (Fig. 4, bottom panels). Only some internal "deformation" of the NP fiducials was detected, most likely because of in vivo evaporation of the aqueous solvent from the AuNP suspensions. Such drawback should be further improved in the future to guarantee more long-term stability, although it can be envisaged that less concentrated fiducials should be less prone to particle sedimentation.

In this work, we designed and fabricated a new class of highly efficient fiducial markers for X-ray imaging and radiosurgery, based on engineered AuNP suspensions encapsulated in PDMS. These devices rely on a smart fabrication strategy that successfully combines AuNP performances, in terms of excellent and tunable X-rays attenuation, with the properties of PDMS, as a biocompatible polymer widely employed for long-term medical implants. In vitro and in vivo evaluations confirmed superior efficiency, durability, and reliability of the NP fiducials that, together with their simplicity and low-cost fabrication, may promise great and wide potential in the expanding field of radiosurgery, e.g. by enabling radiation oncologists with new tools for precise and efficient treatment planning, as exemplified by the pilot data reported in this paper. Moreover, these fiducial markers can be further improved, by optimizing their shape, size, and texture, making them suitable for endoscopic implantation, thus reaching unexplored, clinically relevant body districts by a completely non-invasive approach. Additionally, by exploiting the chemistry of silicone elastomers, it may be possible to further expand the effectiveness and versatility of these fiducial markers, e.g. by loading their shells with biologically-active molecules, also achieving drug delivery purposes. Hence, our investigations represent a preliminary exciting achievement, possibly providing a new route to improve the clinical relevance of radiosurgery, as a primary treatment choice in oncology. 
Acknowledgements

I tipi di Ivrea... bla bla...

Stefano Quarta for useful discussion 

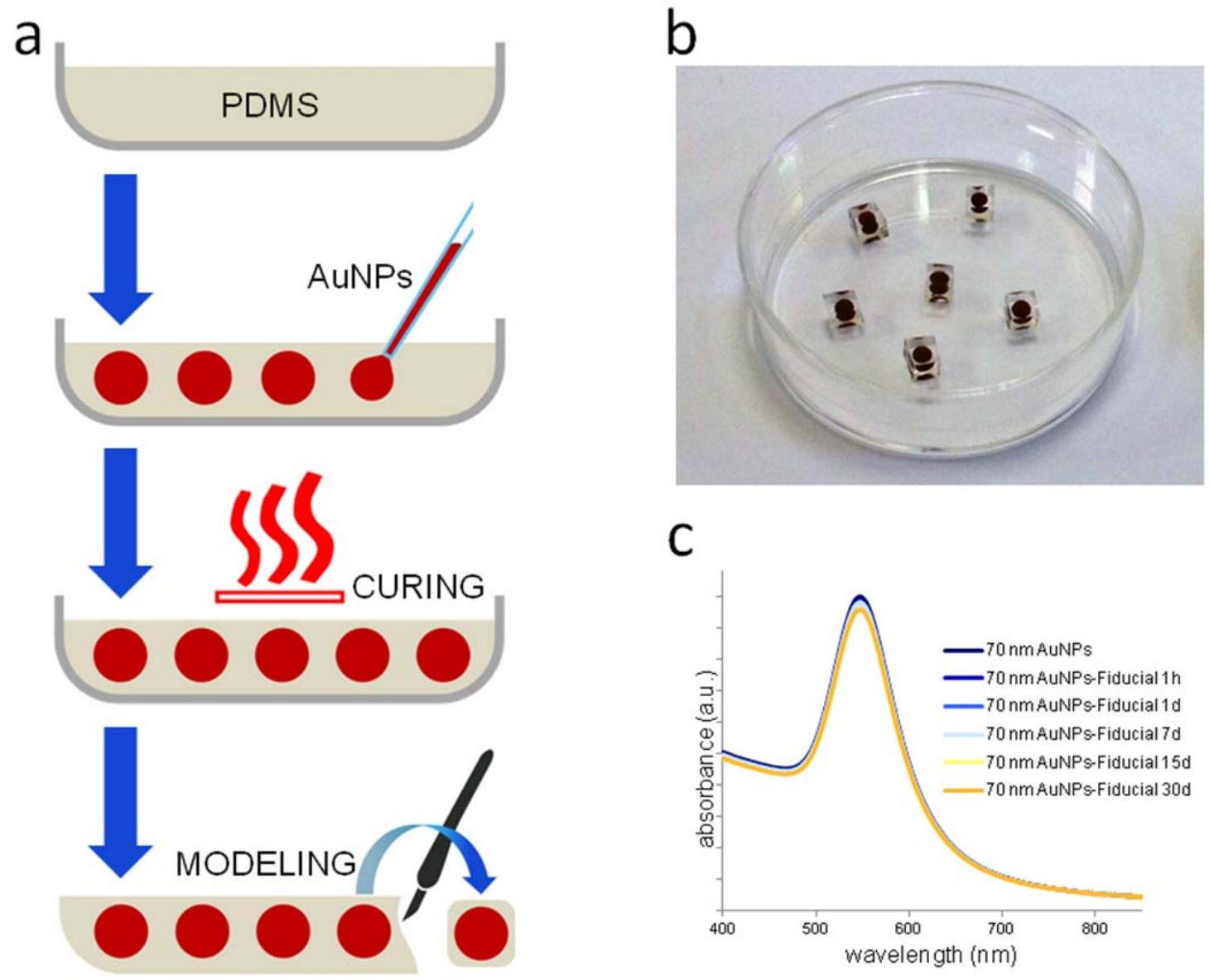

Figure 1. Fabrication of AuNPs-based fiducial markers and stability evaluation overtime. (a) Schematic fabrication procedures of the AuNPs-based fiducial markers by encapsulating AuNPs dispersion in PDMS matrix; (b) image of the prototype fiducial markers with an inner diameter of $1.2 \mathrm{~mm}$ (corresponding to $0.90 \mu \mathrm{l}$ of AuNPs dispersion) in a Petri dish; (c) UV-vis absorption spectroscopy of the AuNPs immediately before encapsulation and during 30 days inclusion into the polymer matrix at ambient conditions. 

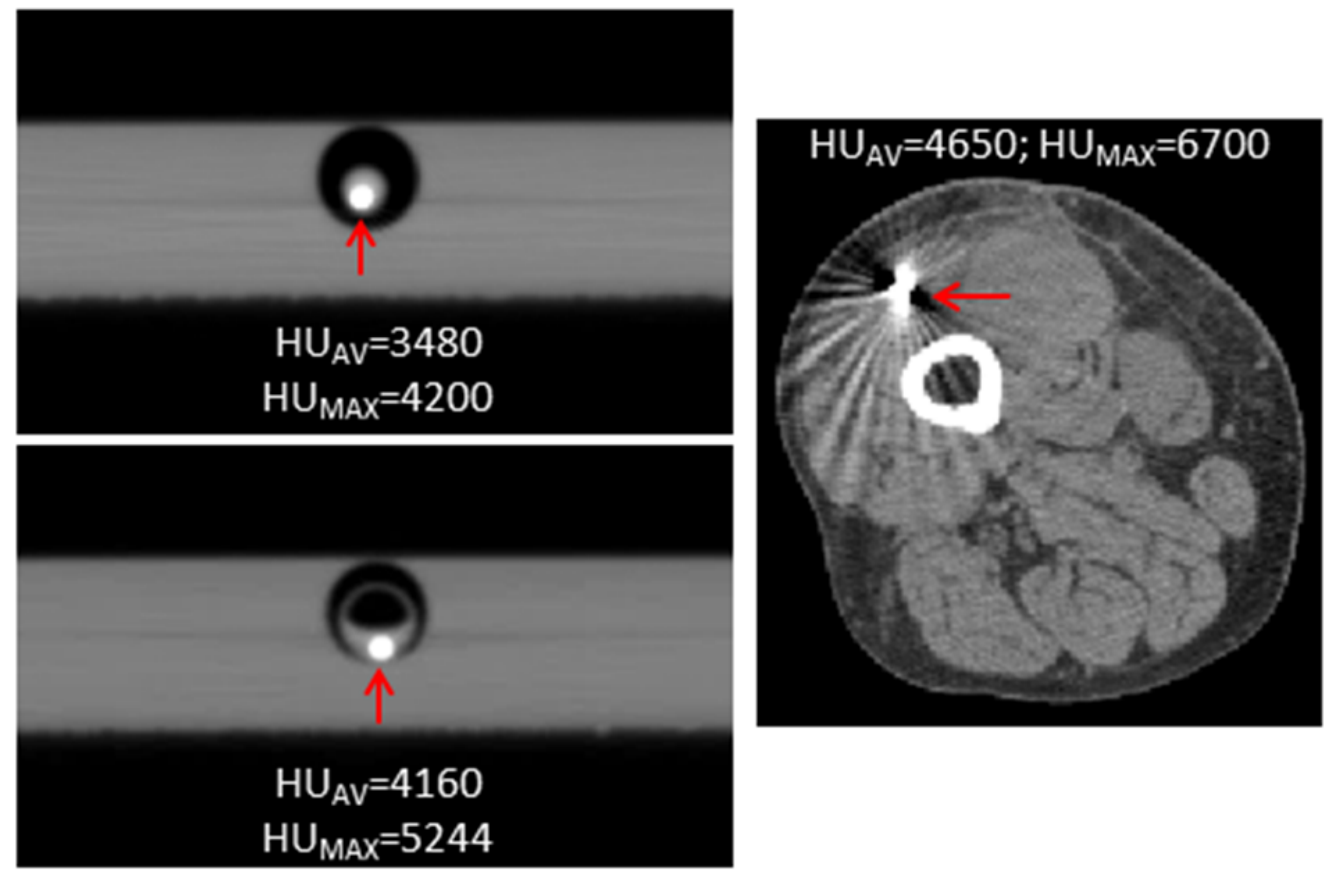

Figure 2. Representative CT images of the NP fiducial markers in tissue-equivalent phantoms (left) and a standard bulk gold fiducial (define dimensions) implanted in a thigh (right). Attenuation efficiency is reported as the averaged and maximum HU measured. Ci vuole un esempio a ca. $10,000 \mathrm{HU}$. 


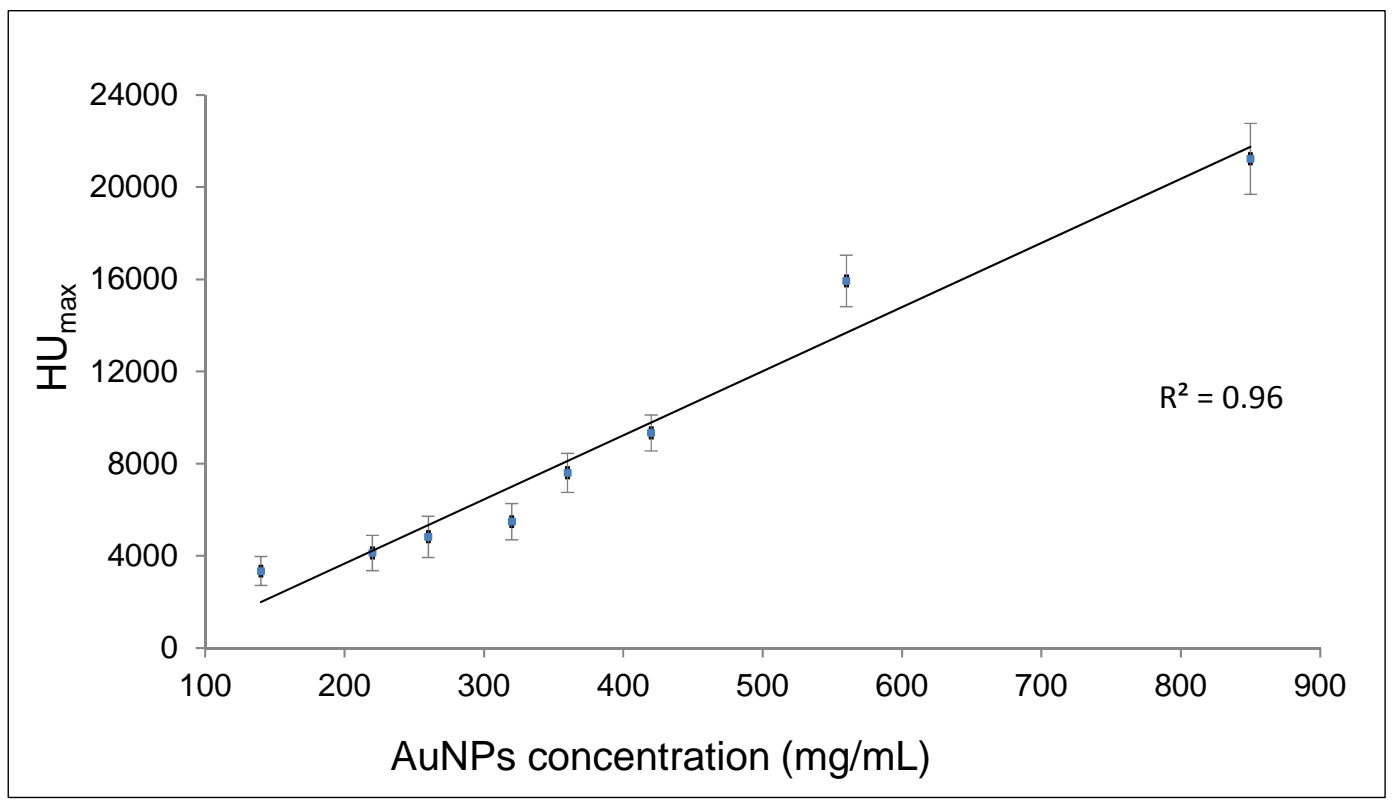

Figure 3. X-rays attenuation efficiency of the NP fiducial markers (expressed as HU units) as a function of AuNPs concentration. Experimental points represent the average of $\mathrm{X}$ independent measurements, while error bars represent the standard deviation. 

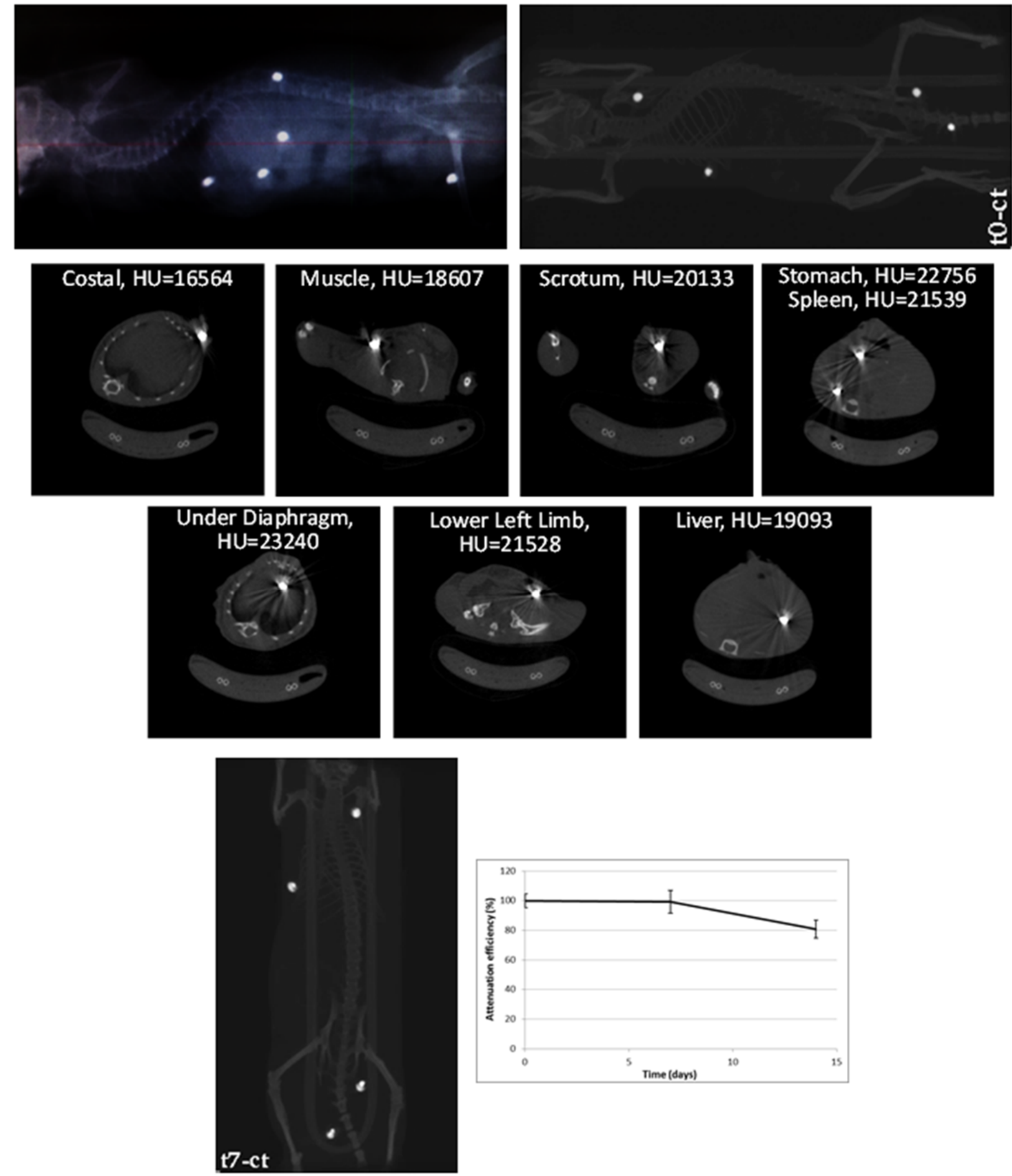

Figure 4. In vivo evaluation of the NP fiducial markers implanted in different body districts in different mice. (Top panels) Representative planar whole-animal CT images of two mice, taken soon after fiducial implantation (left: $1.8 \mathrm{~mm}$ fiducials; right: $1.2 \mathrm{~mm}$ fiducials); (Middle panels) Axial CT images of the fiducials in different organs, with the relative X-ray attenuations recorded; (Bottom panels) Planar CT image of a mouse acquired 1 week after NP fiducials implant (the animal is the same imaged at time zero in the top-right panel), and plot describing the fiducial attenuation efficiency in vivo over-time, averaged on the different fiducial devices. 


\section{Methods}

\section{Gold Nanoparticles synthesis}

All glassware and the magnetic stir-bars were washed thoroughly with aqua regia $\left(\mathrm{HCl}\right.$ and $\mathrm{HNO}_{3}$ in a 3:1 volumetric ratio). First of all, $15 \mathrm{~nm}$ spherical, citrate capped AuNPs were synthesized to be employed as seed by the classical Turkevich-Frens reaction.[29, 30] Briefly, a two-neck round bottom flask was filled with $500 \mathrm{~mL}$ of ultrapure water $(18 \mathrm{M} \Omega \cdot \mathrm{cm}$ in terms of electrical resistivity), then $3 \mathrm{~mL}$ of $0.1 \mathrm{M}$ aqueous solution of HAuCl4 (Sigma-Aldrich) was added to water and the solution was heated to boiling under reflux, while stirring. When boiling started $11.4 \mathrm{~mL}$ of $0.1 \mathrm{M}$ aqueous solution of trisodium citrate (Sigma-Aldrich) was added. The solution was kept gently boiling until a red wine color appeared and then slowly cooled down for the subsequent use. AuNPs of $70 \mathrm{~nm}$ in diameter were prepared by a seed-mediated method which allows the enlargement of $15 \mathrm{~nm}$ AuNPs (seeds) for the property of $\mathrm{NH}_{2} \mathrm{OH}$ to efficiently reduce $\mathrm{Au}^{3+}$ to bulk metal in the presence of $\mathrm{Au}$ surface.[29] The synthesis was performed by adding an appropriate amount of $15 \mathrm{~nm}$ gold seeds into $900 \mathrm{~mL}$ of ultrapure water(18 $\mathrm{M} \Omega \bullet \mathrm{cm})$. The solution was kept under vigorous stirring and then $12 \mathrm{~mL}$ of aqueous $0.1 \mathrm{M}$ hydroxylamine sulfate (Sigma-Aldrich) was added. Subsequently, $40 \mathrm{~mL}$ of $10 \mathrm{mM}$ aqueous solution of $\mathrm{HAuCl}_{4}$ was dropwise added to the seed solution. Stirring was continued for 10 minutes after the addition has ended and then an aqueous solution of HEPES (4-(2-hydroxyethyl)-1-piperazineethanesulfonic acid) (Sigma-Aldrich) $0.1 \mathrm{M}, \mathrm{pH} 7.4$ was injected to a final concentration of $10 \mathrm{mM}$ to stabilize the colloidal solution.

\section{Preparation of the AuNP solution and characterization}

The gold nanoparticles prepared as described above were concentrated by centrifugation. Polycarbonate tubes were filled with AuNP solution and the centrifuged for $40 \mathrm{~min}$ at $1000 \mathrm{~g}$. The supernatant was discarded while the pelleted nanoparticles were recovered. This process was repeated three times to ensure an appropriate nanoparticle concentration. Finally, glycerol (Sigma Aldrich) was added in the colloidal solution to have a glycerol/ $\mathrm{H}_{2} \mathrm{O}$ volume ratio 1 to 2 . The AuNP concentration was measured during each step of concentration by inductively coupled plasma atomic emission spectroscopy (Agilent 720/730 spectrometer). Briefly, samples were dissolved overnight in $1 \mathrm{~mL}$ of aqua regia, diluted to $10 \mathrm{~mL}$ with ultrapure water, and the resulting solution was directly analyzed against standard Au solutions from 0.01 to $10 \mathrm{ppm}(\mathrm{mg} / \mathrm{L})$. The concentration was then adjusted to desired value by adding ultrapure water. Moreover, AuNP dispersions were deeply characterized by several techniques in order to obtain information about size, shape, surface chemistry and dispersion. The 300 mesh carbon-coated copper grid was cast with a few drops of AuNPs and vacuum-dried. TEM images of each sample were collected using a JEOL Jem 1011 
transmission electron microscope with an accelerating voltage of $100 \mathrm{kV}$. By using a Zetasizer Nano-ZS instrument (Malvern Instruments) equipped with a $4.0 \mathrm{mV} \mathrm{HeNe} 633 \mathrm{~nm}$ laser, Dynamic Light Scattering (DLS) analysis revealed the nanoparticle monodispersion and the absence of aggregation or agglomeration phenomena. The same information was obtained in a complementary way by UV-visible spectroscopy. UV-vis spectra were recorded using a Shimadzu UV/Vis/NIR UV3600 at a $300 \mathrm{~nm} / \mathrm{min}$ scanning rate from 350 to $700 \mathrm{~nm}$. Furthermore, the proper surface charge value, that allows nanoparticle colloidal stability by electrostatic repulsion, was calculated by carrying out -potential analysis (Zetasizer Nano-ZS).

\section{AuNPs encapsulation in PDMS matrix}

Sylgard ${ }^{\circledR} 184$ silicon elastomer (Dow Corning) was employed to encapsulate the AuNP dispersion. The elastomer and the curing agent were mixed in a ratio 10:1 following the manufacturer's instructions. The solution was degassed in a vacuum chamber to remove bubbles, after that it was poured in a Petri dish and drops of AuNP dispersion was inserted by a positive displacement micropipette (Eppendorf). By controlling the volume of nanoparticle solution a precise control over the final drop diameter can be achieved. The polymer with AuNP drops inside was heat cured in a hoven for $60 \mathrm{~min}$ at $70^{\circ} \mathrm{C}$. Subsequently, the slab of cured PDMS was removed from the Petri dish and single fiducial markers were easily obtained using a scalpel. The optical properties of the AuNP dispersion after encapsulation were evaluated by slicing the fiducial marker and recovering AuNPs with a micropipette. Then, an appropriate amount of nanoparticle dispersion was diluted in water and subsequently UV-vis spectra were recorded using a Shimadzu UV/vis/NIR UV3600 at a 300 $\mathrm{nm} / \mathrm{min}$ scanning rate from 350 to $700 \mathrm{~nm}$.

\section{Fiducial markers analyses by CT scan (Cristina...)}

AuNPs based fiducial markers fabricated with increasing concentration of AuNPs (100, 350 and $850 \mathrm{mg} / \mathrm{mL}$ ) were inserted into a plastic tube of the phantom and compared with water for CT imaging evaluation. The phantom was imaged under the following conditions: 3D CT imaging with GMI-Triumph Tri-Modality Pre-Clinical scanner at $75 \mathrm{kV}$ with 516 views, 1.3 of magnification $(\mathrm{FOV}=91.1 \mathrm{~mm})$ and $2 \times 2$ binning. Attenuation is reported as Hounsfield Unit (HU).

\section{Animal implantation and CT acquisition}

Four healthy mice were implanted with two different fiducial markers (1.2 and $1.8 \mathrm{~mm}$ of inner diameter). Each fiducial marker was inserted in mice in different position under controlled gas anesthesia. After implantation, all mice received Rimadyl $(5 \mathrm{mg} / \mathrm{kg}$ ) to alleviate post-surgical pain. Then, the mice were individually positioned on a heated bed for the CT scans. CT images were 
recorded over a period of 2 weeks after fiducial implantation to monitor location and efficiency of these devices. The CT images were acquired on GMI-Triumph Tri-Modality Pre-Clinical scanner at $75 \mathrm{kV}$ with 516 views, 1.3 of magnification $(\mathrm{FOV}=91.1 \mathrm{~mm})$ and $2 \times 2$ binning. Attenuation is reported as Hounsfield Unit (HU). 


\section{References}

1. Lawrence TS, Ten Haken RK, Giaccia A. Principles of Radiation Oncology. In: DeVita VT Jr., Lawrence TS, Rosenberg SA, (eds). DeVita, Hellman, and Rosenberg's Cancer: Principles and Practice of Oncology. 8th ed. Philadelphia, Pa: Lippincott Williams and Wilkins, 2008.

2. Washington CM, Leaver DT. Principles and Practice of Radiation Therapy, 3rd edition, Mosby.

3. Bomford K. Radiation Protection, In Bomford CK, and Kunkler IH, editors. Walter and Miller's Textbook of Radiotherapy: Radiation Physics, Therapy and Oncology. 2003. New Yorl: Curchill Livingstone.

4. Bortfeld T. Optimized planning using physical objectives and constraints. Seminars in Radiation Oncology 1999, 9(1):20-34.

5. Mok H., Crane C.H., Palmer M.B., Briere T.M., Beddar S., Delclos M.E., Krishnan S., Das P. Intensity modulated radiation therapy (IMRT): differences in target volumes and improvement in clinically relevant doses to small bowel in rectal carcinoma. Radiation Oncology 2011, 6(63): 1-9.

6. Glatstein E. Intensity-modulated radiation therapy: the inverse, the converse, and the perverse. Seminars in Radiation Oncology 2002, 12(3): 272-281.

7. Balter J.M., Lam K.L., Sandler H.M., Littles J.F., Bree R.L., Ten H.R.K. Automated localization of the prostate at the time of treatment using implanted radiopaque markers: technical feasibility. International Journal of Radiation Oncology Biology Physics 1995, 33(5): 1281-1286.

8. Seppenwoolde Y1, Shirato H, Kitamura K, Shimizu S, van Herk M, Lebesque JV, Miyasaka $\mathrm{K}$. Precise and real-time measurement of $3 \mathrm{D}$ tumor motion in lung due to breathing and heartbeat, measured during radiotherapy. International Journal of Radiation Oncology Biology Physics 2002, 53(4):822-834.

9. Balter JM1, Dawson LA, Kazanjian S, McGinn C, Brock KK, Lawrence T, Ten Haken R. Determination of ventilatory liver movement via radiographic evaluation of diaphragm position. International Journal of Radiation Oncology Biology Physics 2001, 51(1):267-270.

10. Murphy M.J., Adler J.R., Bodduluri M., Dooley J., Forster K., Hai J., Le Q., Luxton G., Martin D., Poen J. Image-guided radiosurgery for the spine and pancreas. Computer Aided Surgery 2000; 5(4): 278-288. 
11. van Asselen B., Dehnad H., Raaijmakers C.P., Lagendijk J.J., Terhaard C.H. Implanted gold markers for position verification during irradiation of head-and-neck cancers: a feasibility study. International Journal of Radiation Oncology Biology Physics 2004, 59(4): 1011-1017.

12. M. R. Moman, U. A. van der Heide, A. N. T. J. Kotte, M. R. J. A. van, G. H. Bol , S. P. G. Franken, M. van Vulpen, Radiother. Oncol. 2010, 96 , 38 .

13. S Gill, J Li, J Thomas, M Bressel, K Thursky, C Styles, K H Tai, G M Duchesne, and F Foroudi. Patient-reported complications from fiducial marker implantation for prostate imageguided radiotherapy. Br J Radiol. Jul 2012; 85(1015): 1011-1017.

14. Delouya G1, Carrier JF, Béliveau-Nadeau D, Donath D, Taussky D. Migration of intraprostatic fiducial markers and its influence on the matching quality in external beam radiation therapy for prostate cancer. Radiother Oncol. 2010 Jul;96(1):43-7. doi: 10.1016/j.radonc.2010.03.017. Epub 2010 Apr 8.

15. Bhagat N1, Fidelman N, Durack JC, Collins J, Gordon RL, LaBerge JM, Kerlan RK Jr. Complications associated with the percutaneous insertion of fiducial markers in the thorax. Cardiovasc Intervent Radiol. 2010 Dec;33(6):1186-91. doi: 10.1007/s00270-010-9949-0. Epub 2010 Jul 27.

16. $\mathrm{Ng} \mathrm{M,} \mathrm{Brown} \mathrm{E,} \mathrm{Williams} \mathrm{A,} \mathrm{Chao} \mathrm{M,} \mathrm{Lawrentschuk} \mathrm{N,} \mathrm{Chee} \mathrm{R.} \mathrm{Fiducial} \mathrm{markers} \mathrm{and}$ spacers in prostate radiotherapy: current applications. BJU Int. 2014 Mar;113 Suppl 2:13-20. doi: 10.1111/bju.12624.

17. Khashab MA, Kim KJ, Tryggestad EJ, Wild AT, Roland T, Singh VK, Lennon AM, Shin EJ, Ziegler MA, Sharaiha RZ, Canto MI, Herman JM. Comparative analysis of traditional and coiled fiducials implanted during EUS for pancreatic cancer patients receiving stereotactic body radiation therapy. Gastrointest Endosc. 2012 Nov;76(5):962-71. doi: 10.1016/j.gie.2012.07.006.

18. Jarraya H1, Chalayer C2, Tresch E3, Bonodeau F2, Lacornerie T4, Mirabel X4, Boulanger T2, Taieb S2, Kramar A3, Lartigau E4, Ceugnart L2. Novel technique for hepatic fiducial marker placement for stereotactic body radiation therapy. Int J Radiat Oncol Biol Phys. 2014 Sep 1;90(1):119-25. doi: 10.1016/j.ijrobp.2014.05.002. Epub 2014 Jun 28.

19. Hassler C, Boretius T, Stieglitz T. Polymers for Neural Implants. JOURNAL OF POLYMER SCIENCE: PART B: POLYMER PHYSICS 2011, 49, 18-33.

20. Ahn S, Jung SY, Lee SJ. Gold nanoparticle contrast agents in advanced X-ray imaging technologies. Molecules. 2013 May 17;18(5):5858-90.

21. J. F. Hainfeld, D. N. Slatkin, T. M. Focella, and H. M. Smilowitz, "Gold nanoparticles: a new X-ray contrast agent,” British Journal of Radiology, vol. 79, no. 939, pp. 248-253, 2006. 
22. D. P. Cormode, T. Skajaa, Z. A. Fayad, and W. J. M. Mulder, "Nanotechnology in medical imaging: probe design and applications," Arteriosclerosis, Thrombosis, and Vascular Biology, vol. 29, no. 7, pp. 992-1000, 2009.

23. C. Xu, G. A. Tung, and S. Shouheng, "Size and concentration effect of gold nanoparticles on X-ray attenuation as measured on computed tomography," Chemistry of Materials, vol. 20, no. 13, pp. 4167-4169, 2008.

24. Li N, Zhao P, Astruc D. Anisotropic gold nanoparticles: synthesis, properties, applications, and toxicity. Angew Chem Int Ed Engl. 2014 Feb 10;53(7):1756-89.

25. Ryan D. Ross, Lisa E. Cole, Jennifer M. R. Tilley, and Ryan K. Roeder. Effects of Functionalized Gold Nanoparticle Size on X-ray Attenuation and Substrate Binding Affinity. Chem. Mater., 2014, 26 (2), pp 1187-1194

26. K. R. Brown and M. J. Natan, Langmuir, 2008, 14, 726.

27. G. Stremsdoerfer, H. Perrot, J. R. Martin and P. J. Clechet, J. Electrochem. Soc., 1988, 135, 2881.

28. N. E. Good, G. D. Winget, W. Winter, T. N. Connolly, K. Izana and R. M. M. Singh, Biochemistry, 1966, 5, 467.

29. J. Turkevich, P. C. Stevenson and J. A. Hillier, Discuss. Faraday Soc., 1951, 11, 55.

30. G. Frens, Nature (London), Phys. Sci., 1973, 241, 20. 